\title{
Le care, une « voix différente » pour l'histoire du genre
}

Care, a "different voice" for gender history

\section{Clyde Plumauzille et Mathilde Rossigneux-Méheust}

\section{(2) OpenEdition \\ 12 Journals}

\section{Édition électronique}

URL : https://journals.openedition.org/clio/16058

DOI : $10.4000 /$ clio. 16058

ISSN : 1777-5299

Éditeur

Belin

\section{Édition imprimée}

Date de publication : 1 juillet 2019

Pagination : 7-22

ISSN : 1252-7017

\section{Référence électronique}

Clyde Plumauzille et Mathilde Rossigneux-Méheust, « Le care, une « voix différente » pour l'histoire du genre », Clio. Femmes, Genre, Histoire [En ligne], 49 | 2019, mis en ligne le 23 mars 2020, consulté le 02 janvier 2023. URL : http://journals.openedition.org/clio/16058 ; DOl : https://doi.org/10.4000/clio. 16058 


\title{
Le care, une « voix différente » pour l'histoire du genre
}

\author{
Clyde PlumauziLle \\ \& Mathilde RossigneUX-MÉHEUST
}

À l'origine de ce dossier, on trouve un mot (et une notion) de l'épistémologie féministe, celui de " care »-terme que nous avons choisi de conserver en anglais tant il est impossible de restituer sa polysémie par un seul terme ou même une expression en français. Popularisé par l'ouvrage In a different voice de la psychologue féministe Carol Gilligan en 1982, le care est à la fois une grille de lecture du monde social et un terrain de recherche inscrit dans les expériences ordinaires des individus ${ }^{1}$. Grille de lecture, le care place la vulnérabilité humaine et sa prise en charge au cœur de l'analyse des rapports moraux et sociaux pour comprendre la façon dont tient et s'entretient une société. Terrain de recherche, le care invite à éclairer les dispositions, les compétences et les activités de soin d'autrui, de souci des autres et les dynamiques sociales et sexuées auxquelles elles s'adossent. Car si le care est résolument une question de genre, c'est aussi une question pour les études de genre. Cette « voix différente» du care est en effet décrite par Gilligan comme l'expression d'une vision du monde sensible, affectée et attentionnée, souvent dévolue aux femmes. C'est précisément parce qu'elle découle d'une division sexuée de la prise en charge d'autrui, historiquement construite, que l'assignation des femmes à la préservation et l'entretien du bien être des individus peut se perpétuer dans la longue durée. Cette assignation déborde cependant la simple opposition hommes/femmes car le care peut être délégué, reporté, acheté ; si certaines femmes arrivent à s'en émanciper, c'est le plus

1 Gilligan 2008 [1982] ; Laugier 2009. 
souvent au prix d'une mise au service d'autres femmes plus précaires, de travailleurs pauvres, ou d'étranger.es. Regarder les rapports sociaux avec les lunettes du care c'est donc aussi affiner le questionnement du genre et, à l'instar de l'intersectionnalité, approfondir la problématique des inégalités entre femmes.

\section{Une catégorie travaillée par l'histoire}

Depuis la fin des années 2000 en France, des chercheurs et surtout des chercheuses en sciences sociales se sont saisis de cette catégorie pour interroger l'émergence contemporaine d'un "grand marché mondial du soin d'autrui », alimenté notamment par les migrations de femmes des pays du Sud dans les métropoles des pays du Nord. Si le care est un enjeu pour décrypter notre présent, il nous paraît également pouvoir proposer des pistes de réflexion et d'analyse pour le passé. Historiennes des femmes et du genre, travaillant sur les rapports de domination qui pèsent sur les populations subalternes et leurs inscriptions dans le corps des individus, nous avons souhaité nous emparer de cette perspective de recherche afin de sonder ses potentialités pour notre discipline. Le dossier que nous proposons est une invitation à faire l'histoire du genre avec le care.

De fait, si les méthodes de l'enquête historique n'ont pas été mobilisées dans le champ des études de care, une place indéniable a été faite dans ces travaux à l'historicité de la prise en charge des besoins d'autrui, à sa féminisation et à sa progressive externalisation et marchandisation. Ce rapport à l'histoire est parfois davantage généalogique. Ainsi Caroline Ibos inscrit-elle l'histoire des nounous ivoiriennes d'aujourd'hui dans le sillage de celle des nounous morvandelles, et finalement dans la perpétuation d'une «histoire injuste, qu'il s'agisse de celle des relations entre les peuples et de celles des relations entre les sexes ». Cet exemple lui permet d'avancer la thèse d'un « retour de la domesticité $»^{2}$ dans nos sociétés européennes. Pour questionner les rapports de pouvoir à l'œuvre dans le travail domestique contemporain et interroger leurs effets psychiques, Pascale Molinier part de l'affaire des Sœurs Papin en 1933, ces «bonnes

2 Ibos 2012. 
meurtrières » dont le geste révèle la complexité des sentiments induits par l'obligation de fournir du care $^{3}$. Convaincues de la richesse de telles opérations généalogiques, nous souhaitons initier un dialogue entre les théories du care et l'histoire des femmes et du genre.

Dans ce dossier en forme d'exploration, les contributions de sept historien.nes et deux sociologues rendent possible cette rencontre. En son cœur, l'état de la recherche proposé par Caroline Ibos offre une cartographie du champ du care mais également une boussole pour qui voudrait s'aventurer sur ce terrain mouvant, récemment institutionnalisé et résolument transdisciplinaire. L'histoire y est à la fois discrète mais omniprésente, tant les études de care s'adossent à une réflexion plus large sur les conditions historiques de la division sociale et morale de la prise en charge des vulnérabilités. Caroline Ibos explique ainsi dans sa contribution au numéro que :

[...] sans recherche historique, sans enquête sociologique, sans terrain ethnographique, la plupart des hypothèses au cœur du care resteraient abstraites et programmatiques, alors même qu'il s'est constitué sur la critique de l'abstraction des éthiques libérales.

Les grandes études sociologiques ou philosophiques envisagent en effet le care dans les processus de transformation du capitalisme (Arlie Hochschild, Nancy Fraser), d'évolution du Welfare (Jane Jenson), de réassignation sexuée des sentiments moraux (Joan Tronto) ou de rapports socio-raciaux (Evelyn Nakano-Glenn, Elsa Dorlin) ${ }^{4}$. Dans leur sillage, on imagine combien l'histoire de la mondialisation, du travail en situation coloniale ou encore de la domesticité gagnera à mobiliser la notion de care pour faire apparaître le rôle de ce type d'activité dans l'émergence d'une société mondialisée.

Ce dossier constitue l'occasion de sortir la problématique du care du très contemporain pour interroger l'évolution des arrangements sociaux du soin entre famille, État, marché, charité ou association, à la faveur de différentes temporalités : la Grèce ancienne, la Provence médiévale, la France et ses territoires colonisés du XIX au XX ${ }^{\mathrm{e}}$ siècle ou encore la Gold Coast britannique des années 1930. Dans son ouvrage Il Welfare prima del Welfare sur l'assistance aux personnes âgées

\footnotetext{
3 Molinier 2005.

4 Hochschild 2013 ; Fraser 2016 ; Jenson 2011 ; Nakano-Glenn 2012 ; Dorlin 2006.
} 
à l'époque moderne, l'historienne Angela Groppi avait déjà souligné la nécessité d'ancrer cette réflexion sur l'évolution des arrangements sociaux du soin dans une chronologie plus large pour déjouer l'idée d'un transfert progressif des devoirs familiaux de care et d'assistance aux institutions publiques et privées. Son étude de la Rome pontificale des $\mathrm{XVI}^{\mathrm{e}}$ et XVII ${ }^{\mathrm{e}}$ siècles décrit au contraire un système de négociations et de transactions constantes entre les individus et diverses institutions charitables pour organiser la prise en charge de la vieillesse. Dans ce numéro de Clio, l'article de Caley McCarthy sur les nourrices de l'Hôpital Saint-Esprit à Marseille à l'époque de la Grande Peste (XIVe siècle) témoigne ainsi de l'existence, à l'échelle municipale, d'un "welfare avant le welfare», c'est-à-dire d'une délégation et d'une institutionnalisation de la prise en charge d'autrui. À travers une étude du salaire des nourrices engagées par l'hôpital, elle montre l'existence d'un travail de care non seulement rémunéré mais aussi valorisé économiquement, d'autant que le travail de ces femmes s'inscrit dans un contexte démographique précaire.

L'histoire contemporaine est néanmoins à l'honneur dans ce dossier. De fait, le XIX siècle constitue un tournant dans l'institutionnalisation du care tant sur le plan de la construction de l'État social que du développement inédit des congrégations féminines à vocation soignante ${ }^{5}$. Ce changement d'échelle dans la mise en ordre du care explique l'abondance archivistique dans les institutions publiques et religieuses, facilitant l'enquête historienne et démultipliant ses possibles. Cependant, cette manne de papiers institutionnels n'est pas la seule ressource des historien.nes du care. Chacun et chacune a également tenté de mobiliser une documentation à hauteur d'individu. Dans l'ensemble des contributions, les auteur.es s'efforcent, notamment pour les périodes antérieures, de traquer le care derrière les mots des acteurs et des actrices du passé qui disent et font le soin et la sollicitude au travail. On soulignera ici l'importance des sources épigraphiques pour l'étude d'Hélène Castelli sur les guérisseuses en Grèce ancienne, celle des sources iconographiques telles les images pieuses analysées par

5 Langlois 1984 ; De Luca Barusse 2002 ; Jusseaume 2016 ; Brejon de Lavergnée 2018 ; Capuano 2018. 
Matthieu Brejon de Lavergnée pour interroger l'intériorisation de la sollicitude chez les Sœurs de la Charité, ou encore celle des égodocuments qui, à l'instar des récits de vie recueillis et des photos de famille exhumées par Violaine Tisseau, peuvent restituer des savoirêtre de care propres à la domesticité coloniale à Madagascar.

\section{Poursuivre l'histoire de la division sexuée du travail}

C'est sous l'angle du travail, et donc du travail de care, que ce numéro propose d'ouvrir les voies d'une histoire du genre avec le care. Nous nous sommes particulièrement appuyées sur les propositions théoriques de la philosophe Joan Tronto qui, dès 1990, a proposé une définition du care comme activité «qui comprend tout ce que nous faisons pour maintenir, perpétuer et réparer notre "monde", de sorte que nous puissions y vivre aussi bien que possible $»^{6}$. Cette formulation du care comme activité plutôt que comme disposition rend possible une réflexion historique et politique portant sur l'organisation et la répartition de cette charge essentielle à la satisfaction des besoins primordiaux. Joan Tronto constate ainsi que c'est généralement aux plus vulnérables qu'est assigné ou délégué le «fardeau » du care. Cette réflexion n'est pas sans rappeler le «débat sur le travail domestique » initié par des intellectuelles féministes à la fin des années 1960 et participe d'une même volonté de mettre en cause l'idée d'une division et d'une hiérarchisation naturelle entre travail productif et travail reproductif ${ }^{7}$. La formulation d'une perspective de care dans le champ des sciences sociales doit alors être ressaisie à l'aune de toute une tradition féministe, notamment historienne.

$\mathrm{Si}$ cette dernière est rarement le référentiel des recherches des sciences sociales, elle résonne pourtant pour nous comme une évidence. C'est en effet de cette «histoire sans qualités », attentive au travail invisible, aux façons de dire et aux façons de faire des femmes, que nous venons ${ }^{8}$. Reprenant le constat de nombreux travaux d'anthropologie sur la dimension quasi universelle de la division par

\footnotetext{
$6 \quad$ Fisher \& Tronto $1991: 40$.

7 Charles \& Galerand 2017.

$8 \quad$ Verdier 1979.
} 
sexes des activités dans les sociétés, des historiennes se sont saisies de ce continuum supposé pour en restituer les temporalités, ainsi que les dynamiques sociales et politiques. Chemin faisant, elles ont contribué à «sauver de l'immense condescendance de la postérité ${ }^{9}$ ménagères, servantes de ferme, cuisinières, femmes de chambre, bonnes à tout faire et autres «femmes toutes mains $»^{10}$. Ces recherches ont notamment restitué une part manquante de notre conception du travail dans les sociétés industrielles de la fin du XVIII siècle au début du XXe siècle : celle, largement féminisée et invisibilisée, de la servitude, du travail domestique et du service ${ }^{11}$. Elles ont souligné la fragilité des partitions théoriques entre sphère publique et sphère privée, et élargi la définition du travail au travail informel, non salarié, non rémunéré, ou non marchand ${ }^{12}$. Fortes de cet héritage historiographique, nous souhaitons nous emparer du care pour prolonger une réflexion historique sur les formes de l'expérience du travail.

Qu'apporte la notion de «care » à l'histoire du travail des hommes et des femmes? La psychosociologue Pascale Molinier, qui a contribué à populariser la notion de «travail de care» en France, propose de regrouper sous cette expression «des activités spécialisées où le souci des autres est explicitement au centre», "des activités domestiques réalisées au sein de la famille et leur délégation » et plus largement «une dimension présente dans toutes les activités de service, au sens où servir, c'est prêter attention à $»^{13}$. Là où la catégorie de «travail domestique» a permis d'appréhender comme une forme de travail gratuit et invisibilisé l'ensemble des tâches exercées par les femmes au sein du foyer - allant de l'éducation des enfants aux relations sexuelles en passant par les tâches ménagères celle du travail de care engage à se saisir plus largement de l'ensemble des activités dévolues à la prise en charge des besoins d'autrui,

\footnotetext{
9 Thompson 2012 [1963] : 79.

10 Sur ces "métiers de femmes" mis en lumière par l'histoire du travail des femmes en France, voir notamment les travaux de Perrot (dir.) 1978 et 1987 ; Fraisse 1979; Fourcaut 1982 ; Knibiehler, Leroux-Hugon, Dupont-Hess \& Tastayre 1984.

11 Sarti 2014.

12 Sarti, Bellavitis \& Martini 2018.

13 Molinier 2010.
} 
gratuites comme rémunérées, à l'intérieur comme à l'extérieur du foyer ${ }^{14}$. La polysémie du terme de care, permet en outre de décliner la pluralité des engagements en jeu, qu'il s'agisse de «caring out» (se soucier de), «taking care of 》 (prendre en charge), « care giving» (prendre soin), « care receiving» (recevoir le soin) ${ }^{15}$.

Au cœur de la perspective de care, il y a la saisie des dimensions relationnelle, intentionnelle et émotionnelle du travail, dimensions quelque peu négligées par la notion de travail domestique. Travailler avec le care peut ainsi contribuer à lier l'histoire du travail à celle des émotions, nouveau territoire-frontière de l'historien.ne pour apprécier jusqu'où se jouent et se déjouent les assignations de genre ${ }^{16}$. Un autre prolongement offert par la perspective du care consiste alors à décloisonner l'histoire du travail des femmes en pensant ensemble des activités économiques et des pratiques sociales liées à la gestion et à l'entretien de la vie et du bien-être.

\section{Histoires du travail de care}

Se trouvent ainsi réunies dans ce numéro des contributions sur la domesticité coloniale, les soins aux pauvres, l'assistance sociale, les premiers secours, la maïeutique, la protection infantile, le nourrissage ou encore le guérissage. Avec l'ensemble de ces articles, nous souhaitons donner à voir quelques-uns des chemins historiques qu'il nous est possible d'emprunter dès lors que l'on pense le travail par la relation qui l'institue et la relation qu'il institue. Le care permet en effet d'être à la croisée de différentes historiographies - l'histoire du soin, l'histoire du travail, l'histoire religieuse et l'histoire de la domesticité -, et dès lors de « déplacer les frontières du travail ${ }^{17}$ en interrogeant plus spécifiquement les activités-frontières dans l'organisation du travail. Les contributions de ce dossier interrogent un continuum d'activités allant du travail informel des guérisseuses à domicile dans l'Antiquité (Hélène Castelli), au travail salarié des sages-

14 Chabaud-Richter, Fougeyrollas-Schwebel \& Sonthonnax 1985 ; Delphy 1978 ; Tabet 2004.

15 Tronto 2009 [1993].

16 Boquet \& Lett 2018.

17 Albert, Plumauzille \& Ville 2017. 
femmes (Maï Le Du) à l'époque très contemporaine, en passant par l'allaitement monnayé des nourrices à l'époque médiévale (Caley McCarthy). Elles font varier les échelles d'analyse selon que ces relations s'exercent dans un cadre familial, communautaire ou institutionnel et interrogent la visibilité des travailleuses de care et la reconnaissance sociale de leurs activités.

À l'intérieur même de leurs contributions, certain.es auteur.es soulignent que ces activités de care sont susceptibles de varier de nature selon leurs contextes de mise en pratique : les guérisseuses à domicile de la Grèce ancienne étudiées par Hélène Castelli peuvent également prodiguer des actes de soin à l'extérieur du foyer, et, pour leur technicité, être rémunérées à ce titre. Se trouvent ainsi remises en question les frontières entre cure (therapeia) et care (epimeleia) qui organisent la hiérarchisation des savoirs et des savoir-faire entre la médecine et le soin. Dans son étude documentaire sur les protestations des femmes médecins affectées aux services de Protection maternelle et infantile en Gold Coast britannique dans les années 1920-1930, Anne Hugon montre une remise en cause de la différence entre care et cure et une dénonciation de l'assignation féminine au care, dans la mise en valeur de compétences professionnelles mêlées à des revendications salariales. Une lutte qui, selon l'anthropologue Maï Le Du dans sa contribution à ce numéro, se rejoue en France dans les années 2000 avec l'intégration des études de la filière maïeutique au sein des facultés de médecine et la promotion par les sages-femmes d'une pratique de care « qui relève de compétences plus que d'évidences ».

Prendre en compte le care, dans le travail et comme un travail, met également en lumière l'évolution genrée de certaines professions et éclaire d'un jour nouveau les attentes sociales liées à certaines activités professionnelles. Dans son article sur la professionnalisation des ambulanciers dans la France du XXe siècle, Charles-Antoine Waneck sonde ainsi les ambiguités d'un travail qui relève de deux domaines d'activités a priori éloignés : celui du transport privé, masculin, populaire et peu reconnu; et celui de l'assistance et des premiers secours, plutôt féminin et reconnu dès lors qu'il s'exerce dans un cadre institutionnel et philanthropique. Ces ambiguités peuvent contribuer à rendre cette activité inclassable, et donc déclassée dans l'organisation du travail 
sanitaire; mais elles offrent aussi des marges de manœuvre aux individus dans leur lutte de reconnaissance. Pour les ambulancières de la Croix-Rouge, la valorisation du sang-froid et de la maitrise technique nécessaires au transport des malades leur permet de sortir du simple rôle de soignante. À l'inverse, pour les ambulanciers privés, la promotion d'une pratique de soins de premier secours et la revendication d'une proximité avec les malades leur permet de ne pas être considérés comme de simples transporteurs. Ces éclairages pluriels et situés questionnent donc la longue durée de ces formes de travail de soin, de servitude et de service à la personne, largement invisibilisées, féminisées et le plus souvent subalternes dans l'organisation du travail.

\section{Entre interdépendances de genre, domination rapprochée et travail émotionnel}

La relation de travail en contexte de care met en présence une somme de vulnérabilités : salariales, sociales et sanitaires. Les dominations complexes de genre, de classe, de race et d'âge qu'elle produit sont au cœur de ce dossier.

Qui dépend de qui dans les activités rémunérées ou gratuites de care? D’une simplicité désarmante, cette question propre aux éthiques de care ancre résolument l'histoire du travail dans une perspective relationnelle. L'étude que fait Hélène Castelli de la communauté de femmes libres et non-libres, toutes pourvoyeuses de care au sein de l'oikos, montre, qu'en pratique, les rapports sociaux tissés au quotidien - notamment autour des activités de soin - mettent constamment au corps à corps ou au coude à coude des individus de statuts très divers. Elle souligne ainsi le lien qui unit les femmes libres assignées au soin, y compris de leur maisonnée, et le corps de leurs esclaves lorsque ces derniers sont malades. Le soin des enfants conduit également à penser les rapports de proximité ordinaire : ceux qu'entretiennent ces mêmes femmes libres avec les femmes esclaves qui les assistent dans cette tâche ainsi que ceux qui se nouent entre les enfants de citoyens et leurs nourrices serviles. Les liens construits par le travail de care sont parfois ponctuels dans le temps, à l'instar du care d'urgence des ambulancières et des ambulanciers. Ils peuvent également jouer dans la durée et se nouer à l'échelle de plusieurs vies. Violaine Tisseau décrit ainsi la place 
centrale qu'occupe affectivement la nourrice (nénène) dans une famille de colons à Madagascar au premier XX $X^{\text {e }}$ siècle, son rôle à l'égard des divers membres de la famille et la perpétuation de sa fonction sur deux générations d'enfants ${ }^{18}$. La durabilité du lien dans le temps est même posée comme une condition nécessaire de l'efficacité du travail des assistantes sociales auprès des familles populaires de l'entre-deuxguerres. Le rayonnement géographique de ces travailleuses sociales, et, avant elles, celui des sœurs soignantes à domicile, permet aussi de penser le poids de ce travail de care dans l'espace urbain et les relations de voisinage.

Au sein des relations de travail, les activités liées au care occupent une place singulière : à la fois très hiérarchiques et très proximales, elles s'inscrivent dans un rapport d'interdépendances particulièrement complexes. Peut-être l'expression de «domination rapprochée», élaborée par Dominique Memmi pour décrire cette « relation sociale plaçant ses protagonistes dans une situation d'interdépendance puissante, renforcée par le fait qu'ils se trouvent retenus de manière quasi continue, en situation de co-présence physique, dans un lieu relativement unique et clos $»^{19}$, est-elle la plus riche pour appréhender ces relations de travail modelées en profondeur par la proximité corporelle des care givers et des care receivers. Ce face-à-face est ainsi présent dans toutes les activités des ambulancier.ères étudié.es par Charles-Antoine Wanecq - du brancardage à la mise en condition dans le véhicule, en passant par l'aide aux démarches administratives à l'entrée à l'hôpital et les marques d'attention et de réconfort à la personne accidentée. Dans le temps de l'urgence, apparaît en outre une forme d'égalité dans la dépendance à l'égard des services ambulanciers. Cette temporalité exceptionnelle permet alors de penser la singularité de ce rapport au care, susceptible, de la mise en nourrice ou à la crèche jusqu'à la mise en bière, de concerner l'ensemble du corps social ${ }^{20}$.

Inscrire le travail de care proposé, fourni ou vendu par un care giver dans un rapport de dépendance socialement redistribué ne revient cependant pas à gommer les rapports de domination. Les articles

\footnotetext{
18 Sur ce point voir également Drouilleau-Gay 2019.

19 Memmi 2008 et 2016.

20 Memmi 2017.
} 
présentés dans ce dossier montrent la très grande diversité des situations et l'intérêt de penser le travail de care à l'intersection des dominations de classe, de genre, d'âge et de race. Et l'expression de D. Memmi semble parfaitement taillée pour décrire la relation de travail dans laquelle s'inscrivent domestiques, esclaves ou encore nourrices directement placé.es sous l'autorité des maître.sses de maison. La hiérarchie qui façonne le travail des sœurs soignantes s'avère autrement complexe, puisqu'il est pris entre, d'une part, la domination des administrateurs, du corps médical et des bienfaiteurs, et d'autre part, la dépendance quotidienne des malades. Enfin contrairement aux travailleuses de care étudiées depuis vingt ans par les sociologues, les sœurs, les ambulancières de la Croix-Rouge ou les assistantes sociales présentes dans ces articles n'occupent pas une position subalterne dans l'espace social. Pour elles, faire profession du soin des autres constitue au contraire une trajectoire attendue pour des femmes presque toutes issues de milieux privilégiés ; leur travail au contact des classes populaires les place en situation évidente de domination sociale. Les voies ouvertes par les observatrices du travail de care contemporain invitent à ne pas présupposer la nature des dépendances qui structurent toutes les activités liées au care. Les travaux en sociologie d'Eve Meuret-Campfort, Pascale Molinier ou de Christelle Avril montrent ainsi que la plus vulnérable est a priori la personne aidée, mais que la travailleuse est largement fragilisée par la précarité de ses conditions de travail, et ce, d'autant plus que l'éthique du care l'enjoint à s'oublier devant les intérêts de l'autre ${ }^{21}$. On pourrait ainsi multiplier les exemples, mais retenons seulement qu'observer le travail de care constitue une invitation sans cesse renouvelée à interroger le processus de construction des dominations à l'œuvre dans le cadre d'un travail largement féminisé.

Analyser la nature, l'intensité et surtout l'expression des émotions à l'œuvre dans les relations de care est centrale pour souligner la singularité de ces professions liées au care. Le care invite en effet à traquer dans les activités de service et de soin ce que la sociologue Arlie Hochschild a dénommé le «travail émotionnel», à savoir la compréhension, l'évaluation et la gestion des émotions, les siennes et

21 Molinier 2006 et 2009 ; Avril 2014 ; Meuret-Campfort 2014 et 2017. 
celles d'autrui ${ }^{22}$. Le rapport professionnel peut par exemple donner lieu à une relation affective : à la mort de la servante, la maitresse évoquée par Hélène Castelli lui fait dresser une stèle personnalisée, élaborée et coûteuse, qui met en scène l'activité de care et réunit à jamais les deux femmes dans la mort. Les odeurs liées au portage des nourrissons et leur mise en souvenir montrent aussi, selon Violaine Tisseau, combien la relation de care est inscrite dans les émotions du corps. En outre, la puissance et la continuité du discours vocationnel dans l'univers des professionnelles du care (sœurs, femmes médecins en mission, assistantes sociales et ambulancières de la Croix-Rouge) placent d'emblée et pour des raisons morales, leur travail dans le monde des affects. Au-delà de l'injonction à la vocation directement issu de la sphère religieuse et charitable, des recommandations sont formulées à mesure que les professions s'institutionnalisent pour clarifier la nature même des émotions à éprouver et à mettre en scène dans le cadre du travail. Dans son article sur les premières générations d'assistantes sociales dans l'entre-deux-guerres, Lola Zappi, emboitant le pas aux travaux fondateurs de la sociologue Beverley Skeggs, esquisse une histoire des émotions formalisées et valorisées dans la formation au travail social, histoire où le vocabulaire du lien familial cède du terrain à celui de l'amitié ${ }^{23}$. À la recherche de la «bonne distance», les ouvrages théoriques de l'époque encouragent ainsi ces travailleuses du care à façonner les répertoires affectifs sur lesquels elles peuvent jouer auprès des familles populaires. Dans un autre registre, les images pieuses mettant en scène les sentiments filiaux des Sœurs de la Charité présentées par Matthieu Brejon de Lavergnée contribuent à une plus grande diffusion de la dimension affective du travail des sœurs et à sa standardisation. Les articles dans ce dossier permettent de mettre en avant le poids de l'histoire contemporaine du travail de care dans l'essentialisation des qualités émotionnelles supposées féminines, tant convergent la mise en scène et la mise en pratique d'émotions de plus en plus médiatisées, standardisées, formalisées et rémunérées.

Ce dossier est un pari, celui de faire se rencontrer l'histoire du genre et l'épistémologie de care. Pour cela, une réflexion collective déclinée

\footnotetext{
22 Hochschild 1983 et 2017.

23 Skeggs 2015 [1997].
} 
sur plusieurs séquences historiques nous a paru nécessaire pour mettre à l'épreuve le care sur le terrain de l'histoire. Restituant la part sensible des rapports sociaux, questionnant les dynamiques de pouvoir qui les organisent selon les sexes et les positions des individus, les éclairages offerts par les auteur.es de ce numéro poursuivent l'entreprise féministe de dénaturalisation du sexe et offrent de nouveaux territoires à l'histoire sociale des femmes et du genre.

\section{Bibliographie}

Albert Anaïs, Plumauzille Clyde et Sylvain VilLe, 2017, «Déplacer les frontières du travail», Tracés. Revue de Sciences bumaines [En ligne], 32 [http://journals.openedition.org/traces/6822]

Avril Christelle, 2014, Un autre monde populaire. Les aides à domicile, Paris, La Dispute, coll. «Essais ».

Boquet Damien \& Didier LeTt, 2018, «Les émotions à l'épreuve du genre », Clio. Femmes, Genre, Histoire, 47, p. 7-22.

Brejon de Lavergnée Matthieu, 2018, Le Temps des cornettes. Histoire des Filles de la Charité (XIX'-XX'e siècle), Paris, Fayard.

CAPUANO Christophe, 2018, Que faire de nos vieux? Une histoire de la protection sociale de 1880 à nos jours, Paris, Les Presses de Sciences Po.

Chabaud-Richter Danièle, Fougeyrollas-Schwebel Dominique \& Françoise Sonthonnax, 1985, Espace et temps du travail domestique, Paris, La librairie de Méridiens.

Charles Aline \& Elsa Galerand, 2017, «Du travail ménager au service domestique, de l'emploi féminin au travail militant: critiques féministes en évolution ", Recherches féministes, 30/2, p. 1-16.

DALY Mary \& Jane LEWIS, «Conceptualising social care in the context of welfare state restructuring", in Jane LEWIS (ed.), Gender, Social Care and Welfare State Restructuring in Europe, Ashgate, Aldershot, p. 1-24.

DelPhy Christine, 1978, "Travail ménager ou travail domestique?", in Andrée Michel (éd.), Les Femmes dans la société marchande, Paris, Presses universitaires de France, p. 39-54.

De Luca Barusse Virginie, 2002, Aux Origines de l'état providence. Les inspecteurs de l'Assistance publique et l'aide sociale à l'enfance (1820-1930), Paris, INED éditions. 
Dorlin Elsa, 2006, La Matrice de la race. Généalogie sexuelle et coloniale de la nation française, Paris, La Découverte.

Drouilleau-Gay Félicie, 2019, Secrets de familles. Parenté et emploi domestique à Bogotà (Colombie, 1950-2010), Paris, Éditions Pétra.

Fischer Berenice \& Joan TronTO, 1991, "Toward a feminist theory of caring ", in Emily K. ABel \& Margaret K. NeLSON (eds), Circles of Care: work and identity in women's lives, Albany, New York, State University of New York Press, p. 35-62.

Fourcaut Annie, 1982, Femmes à l'usine: ouvrières et surintendantes dans les entreprises françaises de l'entre-deux-guerres, Paris, François Maspero.

FrAISSE Geneviève, 1979, Femmes toutes mains. Essai sur le service domestique, Paris, Le Seuil.

Frazer Nancy, 2016, "Contradictions of capital and care», New Left Review, 100, p. $99-117$.

Gilligan Carol, 2008, Une voix différente, Paris, Flammarion, coll. «Champs essais ». [trad. de In a Different Voice, Cambridge Mass., Harvard University Press, 1982].

HochsCHILD Arlie R., 1983, The Managed Heart: commercialization of buman feeling, Berkeley, University of California Press.

—, 2013, "Éthique du care et capitalisme », in Carol Gilligan, Arlie Hochschild \& Joan Tronto (dir.), Contre l'indifférence des privilégiés. À quoi sert le care, Paris, Payot, p. 69-37.

—, 2017, Le Prix des sentiments. Au caur du travail émotionnel, Paris, La Découverte.

IBOS Caroline, 2012a, Qui gardera nos enfants? Les nounous et les mères, Paris, Flammarion.

—, 2012b, « La mondialisation du care. Délégation des tâches domestiques et rapports de domination », Métropolitiques [https://www.metropolitiques.eu/Lamondialisation-du-care.html]

JENSON Jane, 2011, «Politiques publiques et investissement social: quelles conséquences pour la citoyenneté sociale des femmes?", Cabiers du Genre, 3, hors-série $n^{\circ} 2$, p. 21-43.

Jusseaume Anne, 2016, "Soin et société dans le Paris du XIX siècle. Les congrégations religieuses féminines et le souci des pauvres ", thèse de doctorat en histoire, sous la dir. de Jean-François CHAnet et de Philippe Boutry, Institut d'études politiques de Paris.

Knibiehler Yvonne, Leroux-Hugon Véronique, Dupont-Hess Odile \& Yolande TASTAYre, 1984, Cornettes et blouses blanches. Les infirmières dans la société française (1880-1980), Paris, Hachette.

LANGLOIS Claude, 1984, Le Catholicisme au féminin. Les congrégations françaises à supérieure générale au XIXe siècle, Paris, Éditions du Cerf. 
LAUGIER Sandra, 2009, "L'éthique comme politique de l'ordinaire », Multitudes, 2/3738 , p. $80-88$.

Memmi Dominique, 2008, "Mai 68 ou la crise de la domination rapprochée », in Boris Gobille, Frédérique Matonti \& Bernard Pudal (dir.), Mai-juin 68, Paris, Les Éditions de l'Atelier, p. 35-46.

—, 2016, «Aides à domicile et domination rapprochée », La Vie des idées, 4 mai 2016, [https://laviedesidees.fr/Aides-a-domicile-et-domination-rapprochee.html]

—, 2017, «Care, stigmatisation sociale et femmes : un lien inexorable ? Ou : quand le cadavre se dissout dans le "relationnel" ", Sociétés contemporaines, 105/1, p. 5-29.

MEURET-CAMPfort Eve, 2014, "Dire la pénibilité du travail en crèche? Une enquête auprès d'auxiliaires de puériculture syndicalistes ", Sociétés contemporaines, $95 / 3$, p. 81-108.

_ , 2017, « Il n'est jamais trop tard pour devenir employeur. Les particuliers employeurs âgés et leurs assistantes de vie », Genèses, 106/1, p. 50-71.

Molinier Pascale, 2005, «De la condition de bonne à tout faire au début du $\mathrm{XX}^{\mathrm{e}}$ siècle à la relation de service dans le monde contemporain : analyse clinique et psychopathologique », Travailler, 13/1, p. 7-33.

—, 2006, "Le "care" à l'épreuve du travail. Vulnérabilités croisées et savoir-faire discrets ", in Patricia PAPerman \& Sandra LAugier (dir.), Le Souci des autres. Éthique et politique du Care, Paris, Éditions de l'EHESS, p. 299-316.

—, 2009, "Vulnérabilité et dépendance: de la maltraitance en régime de gestion hospitalière », in Marlène JOUAN (dir.), Comment penser l'autonomie? Entre compétences et dépendances, Paris, Presses universitaires de France, p. 433-458.

_ , 2010, «Au-delà de la féminité et du maternel, le travail du care », Champ psy, 58/2, p. 161-174.

—, 2013, Le Travail du care, Paris, La Dispute, coll. « Le genre du monde ».

Nakano-Glenn Evelyn, 2012, Forced to Care, Coercion and Caregiving in America, Cambridge, Harvard University Press.

Perrot Michelle (dir.), 1978, «Travaux de femmes dans la France du XIXe siècle », Le Mouvement Social, 105.

—, (dir.), 1987, « Métiers de femmes », Le Mouvement Social, 140.

SARTI Raffaella, 2014, "Historians, social scientists, servants, and domestic workers: fifty years of research on domestic and care work ", International Review of Social History, 59/2, p. 279-314.

SARTi Raffaella, Bellavitis Anna \& Manuela Martini, 2018, What is work? Gender at the Crossroads of Home, Family, and Business from the Early Modern Era to the Present, New York, Berghahn Book. 
SkegGs Beverley, 2015 [1997], Des Femmes respectables. Classe et genre en milieu populaire, Marseille, Agone [trad. par M.-P. Pouly de Formations of Class and Gender: Becoming Respectable, Thousand Oaks, Sage, 1997].

TABET Paola, 2004, La Grande arnaque. Sexualité des femmes et échange économico-sexuel, Paris, L'Harmattan.

Thompson Edward P., 2012 [1963], La Formation de la classe ouvrière anglaise, Paris, Le Seuil [trad. par G. Dauvé, M. Golaszewski \& M.-N. Thibault, The Making of the English Working Class, London, Victor Gollancz, 1963].

Tronto Joan, 2009 [1993], Un monde vulnérable. Pour une politique du care, Paris, La Découverte.

VERDIER Yvonne, 1979, Façons de dire, façons de faire. La laveuse, la couturière, la cuisinière, Paris, Gallimard. 